\title{
Elevated Lipoprotein(a) has Incremental Prognostic Value in Type 2 Diabetic Patients with Symptomatic Coronary Artery Disease
}

\author{
Sung Woo Kwon ${ }^{1,2}$, Jong-Youn Kim³ ${ }^{3}$, Ji Min Sung ${ }^{4}$, Young Won Yoon ${ }^{3}$, Ji-Hyuck Rhee ${ }^{5}$, \\ Byoung Kwon Lee ${ }^{3}$, Young-Hak Jung ${ }^{3}$, Ki-Hyun Kim³ ${ }^{3}$, Jung-Joon $\mathrm{Cha}^{3}$, Jong-Kwan Park ${ }^{6}$, \\ Eui-Young $\mathrm{Choi}^{3}$, Bum-Kee Hong ${ }^{3}$, Se-Joong Rim ${ }^{3}$ and Hyuck Moon Kwon ${ }^{3}$ \\ Sung Woo Kwon and Jong-Youn Kim contributed equally to this work.
}

\begin{abstract}
${ }^{1}$ Division of Cardiology, Department of Internal Medicine, Inha University Hospital, Incheon, Republic of Korea
${ }^{2}$ Department of Medicine, Yonsei University Graduate School, Seoul, Republic of Korea

${ }^{3}$ Department of Cardiology and Cardiovascular Center, Gangnam Severance Hospital, Yonsei University College of Medicine, Seoul, Republic of Korea

${ }^{4}$ Graduate School of Health and Welfare CHA University, Seongnam, Republic of Korea

${ }^{5}$ Division of Cardiology, Department of Internal Medicine, Yongin Severance Hospital, Yonsei University College of Medicine, Yongin, Republic of Korea

${ }^{6}$ Division of Cardiology, Department of Internal Medicine, NHIC Ilsan Hospital, Goyang, Republic of Korea
\end{abstract}

\begin{abstract}
Aim: In addition to type 2 diabetes, an elevated $\mathrm{Lp}(\mathrm{a})$ level is known to be a surrogate biomarker of cardiovascular disease. However, recent studies have demonstrated that the $\operatorname{Lp}(\mathrm{a})$ levels are lower in type 2 diabetic patients than in non-diabetic subjects. Therefore, we sought to evaluate the prognostic value of elevated lipoprotein(a) $[\mathrm{Lp}(\mathrm{a})]$ in type 2 diabetic patients with symptomatic coronary artery disease (CAD).

Methods: A total of 1494 diabetic patients with CAD (62.3\% men, mean age: $63.5 \pm 10.3$ years) were enrolled. CAD was diagnosed using invasive coronary angiography, and laboratory values for lipid parameters, including $\operatorname{Lp}(\mathrm{a})$, were obtained on the day of coronary angiography. The patients were divided into tertile groups according to the individual $\mathrm{Lp}$ (a) level. The baseline characteristics, coronary angiographic findings, duration of follow-up and major adverse cardiovascular events (MACEs) were recorded.

Results: Over a mean follow-up period of $4.4 \pm 2.6$ years, there were 59 MACEs (35 cardiac deaths and 24 cases of non-fatal myocardial infarction), for an event rate of $3.9 \%$. A survival probability plot according to the $\operatorname{Lp}(\mathrm{a})$ tertile revealed that an elevated $\mathrm{Lp}(\mathrm{a})$ level was associated with a worse prognosis $(p=0.008)$, after adjusting for age, gender, hypertension, hyperlipidemia, smoking and the extent of CAD. Furthermore, the addition of an elevated $L p(a)$ level to the reference model improved the integrated discrimination improvement $(0.0216, p<0.001)$, continuous net reclassification improvement (NRI) $(0.5721, p=0.012)$ and NRI $(0.1549, p=0.004)$ values.

Conclusions: In terms of the prognosis, elevated $\mathrm{Lp}(\mathrm{a})$ is associated with worse outcomes in type 2 diabetic patients with symptomatic CAD. Furthermore, an elevated $\mathrm{Lp}(\mathrm{a})$ level has incremental prognostic value in type 2 diabetic patients with symptomatic CAD.
\end{abstract}

J Atheroscler Thromb, 2015; 22: 527-534.

Key words: Lipoprotein(a), Diabetes, Coronary artery disease, Prognosis, Adverse cardiac events

\section{Introduction}

Patients with type 2 diabetes present with a metabolic profile different from that of the general popu- lation and exhibit a risk of cardiovascular events twoto four-fold higher than non-diabetic patients ${ }^{1,2)}$. In addition to type 2 diabetes, an elevated lipoprotein(a) $[\mathrm{Lp}(\mathrm{a})]$ level has been recognized to be a cardiovascu- 
lar risk factor in the general population ${ }^{3,4)}$. Furthermore, a recent meta-analysis of 36 prospective studies provided support for the probable causal role of an elevated $L p(a)$ level in the development of cardiovascular disease in the general population ${ }^{5)}$. Although a cross-sectional study reported that type 2 diabetic patients with coronary heart disease have higher Lp(a) levels than those without coronary heart disease, it remains unclear whether an elevated $\mathrm{Lp}$ (a) level causally affects cardiovascular risks in type 2 diabetic patients ${ }^{6}$. Additionally, a recent study demonstrated that the $\mathrm{Lp}(\mathrm{a})$ levels are lower in type 2 diabetic patients than in non-diabetic subjects, further complicating the association between $\mathrm{Lp}(\mathrm{a})$ and cardiovascular risks in this group ${ }^{7)}$. Therefore, we sought to evaluate the prognostic value of elevated $\mathrm{Lp}(\mathrm{a})$ in type 2 diabetic patients with symptomatic coronary artery disease $(\mathrm{CAD})$.

\section{Materials and Methods}

\section{Study Design and Patient Selection}

A total of 1,494 diabetic patients with symptomatic CAD (62.3\% men, mean age: $63.5 \pm 10.3$ years) were enrolled from 2000 to 2010 at Gangnam Severance Hospital (Seoul, Korea). CAD was diagnosed using invasive coronary angiography, and laboratory values for lipid parameters, including the Lp(a) level, were obtained on the day of coronary angiography, with the analyses performed shortly after sampling. Baseline risk factors, coronary angiographic findings, length of follow-up and major adverse cardiovascular events (MACEs), including cardiac death and nonfatal myocardial infarction (MI), were recorded. Institutional review committee approval and informed consent was obtained.

\section{Definition of Variables}

Risk factors were recorded as categorical variables in all patients. Hypertension was defined as a systolic blood pressure of $\geq 140 \mathrm{mmHg}$ and/or diastolic blood pressure of $\geq 90 \mathrm{mmHg}$ or use of antihypertensive agents. Type 2 diabetes was defined as the use of hypoglycemic agents or insulin, a fasting plasma glucose level of $\geq 126 \mathrm{mg} / \mathrm{dl}$, glycosylated hemoglobin (HbA1c) level of $\geq 6.5 \%$ or known but untreated hyperglyce-

Address for correspondence: Hyuck Moon Kwon, Department of Cardiology and Cardiovascular Center, Gangnam Severance Hospital, Yonsei University College of Medicine, 612 Eonju-ro, Gangnam-gu, Seoul, 135-720, Republic of Korea E-mail: kwonhm@yuhs.ac

Received: April 14, 2014

Accepted for publication: October 9, 2014 mia. The homeostasis model assessment insulin resistance index (HOMA-IR) was calculated as follows:

[fasting insulin $(\mu \mathrm{IU} / \mathrm{mL}) \times$ fasting plasma glucose $(\mathrm{mmol} / \mathrm{L})] / 22.5$

A patient was considered to be a smoker, if he/ she currently smoked or had smoked up until one month prior to the baseline coronary angiography examinations. Non-obstructive $\mathrm{CAD}$ was defined as $<50 \%$ luminal narrowing, whereas obstructive CAD was defined as $\geq 50 \%$ luminal narrowing. The extent of obstructive CAD was categorized based on the number of vessels involved (1,2 or 3).

\section{Measurement of Lipid Parameters, Including Lp(a)}

The total cholesterol, high-density lipoprotein (HDL) cholesterol, triglyceride and low-density lipoprotein (LDL) cholesterol levels were measured directly with colorimetric and turbidimetric assays using an autoanalyzer (Beckman Coulter AU5800). Hyperlipidemia was defined as a total cholesterol level of $\geq 240 \mathrm{mg} / \mathrm{dl}$, LDL cholesterol level of $\geq 130 \mathrm{mg} / \mathrm{dl}$, HDL cholesterol level of $<40 \mathrm{mg} / \mathrm{dl}$, triglyceride level of $\geq 200 \mathrm{mg} / \mathrm{dl}$ and/or the use of lipid-lowering medications. $\mathrm{Lp}(\mathrm{a})$ measurement was performed according to the latex agglutination method with an anti-human $\mathrm{Lp}$ (a) monoclonal antibody using a commercial kit [Lp(a) Latex Daiichi] obtained from Daiichi Pure Chemicals Co., Ltd. with an autoanalyzer (Hitachi 7600-110) that is not affected by Apo(a) isoform variation. The patients were divided into three groups according to the respective $\operatorname{Lp}(\mathrm{a})$ concentration: 1) T1: lower tertile group [Lp(a) median value: $4.7 \mathrm{mg} /$ dl (range: $0-8.5)], 2$ ) T2: middle tertile group [Lp(a) median value: $13.5 \mathrm{mg} / \mathrm{dl}$ (range: $8.6-20.7$ )] and 3) T3: upper tertile group [Lp(a) median value: $38.8 \mathrm{mg} /$ dl (range: 20.8-218.0)].

\section{Endpoint Determination and Follow-Up Data Acquisition}

MACE was defined as cardiac death or non-fatal MI. In order to avoid treatment bias, coronary artery revascularization procedures, such as coronary artery bypass graft surgery (CABG) and percutaneous coronary intervention, were not regarded as endpoints ${ }^{8)}$. In patients with multiple cardiovascular events, only the first event was considered for the analysis. Cardiac death was defined as death due to acute MI, ventricular arrhythmia, refractory heart failure or cardiogenic shock. The diagnosis of non-fatal MI required the presence of at least two of the three following criteria: characteristic chest pain, elevated cardiac enzymes or electrocardiographic alterations indicative of MI. Patient follow-up data were collected using an elec- 
tronic medical record review and/or standardized telephone interviews.

\section{Statistical Analysis}

Continuous data were expressed as the mean \pm standard deviation. In cases of skewed data, median values with interquartile ranges were used. All categorical data are presented as a percentage or absolute number. Analyses of variance (ANOVA) and the $\chi^{2}$ test were used to assess differences between three groups. The Cochran-Mantel-Haenszel test was used to analyze the correlation between the $\mathrm{Lp}$ (a) level and extent of CAD. Cumulative event rates as a function of time were estimated according to the Kaplan-Meier method, and survival curves for MACE were compared using the log-rank test. Univariate and multivariate Cox regression analyses were performed to identify associations between the clinical characteristics (age, gender, hypertension, smoking and hyperlipidemia), lipid parameters, Lp(a), extent of CAD and clinical outcomes. Hazard ratios (HRs) were calculated as an estimate of the risk associated with a particular variable with $95 \%$ confidence intervals (CIs) based on binomial distributions. In order to evaluate the incremental value of $\mathrm{Lp}(\mathrm{a})$ in predicting MACE, two models were constructed: 1) Model I: reference model including age, gender, hypertension, smoking, hyperlipidemia and extent of CAD; and 2) Model II: all of the variables for Model I with the addition of $\mathrm{Lp}(\mathrm{a})$. In order to discriminate whether an elevated $\mathrm{Lp}$ (a) level has incremental prognostic value for the prediction of MACE, the likelihood ratio, C-statistics, integrated discrimination improvement (IDI) and net reclassification improvement (NRI) were compared between the two models ${ }^{9,10)}$. All analyses were performed using the SPSS version 15.0 (SPSS, Chicago, IL, USA) and SAS version 9.1.3 (SAS, Cary, NC, USA) software programs. A p-value of less than 0.05 was considered to be statistically significant.

\section{Results}

Of the total 1,494 patients in the study, $62.3 \%$ were men, and the mean age was $63.5 \pm 10.3$ years. The median $\operatorname{Lp}(\mathrm{a})$ level for the overall population was $13.5 \mathrm{mg} / \mathrm{dl}$ (interquartile range: 6.7, 26.3), while that for the T1, T2, and T3 groups was 4.7, 13.5 and 38.8 $\mathrm{mg} / \mathrm{dl}$, respectively. The baseline, laboratory and angiographic characteristics of the study population according to the $\operatorname{Lp}(\mathrm{a})$ tertile groups are summarized in Table 1. The patients with elevated $\mathrm{Lp}(\mathrm{a})$ levels tended to be older $(p<0.0001)$, female $(p=0.004)$ and non-smokers $(p=0.002)$ (Table 1). More patients with an elevated $\operatorname{Lp}(\mathrm{a})$ level had acute coronary syndrome as a clinical presentation $(p=0.014)$ (Table 1), and an elevated $\mathrm{Lp}(\mathrm{a})$ level was found to be associated with higher rates of both obstructive CAD $(p<$ $0.0001)$ and three-vessel obstructive CAD $(p<$ $0.0001)$ at the time of baseline coronary angiography (Table 1). Consequently, the rates of coronary revascularization and CABG were higher in the patients with an elevated $\mathrm{Lp}(\mathrm{a})$ level (Table 1). The total $(p=0.001)$ and LDL $(p<0.0001)$ cholesterol levels were higher in the group with an elevated Lp(a) level, whereas the triglyceride levels $(p=0.012)$ were higher in the group with a normal $\mathrm{Lp}(\mathrm{a})$ level (Table 1). In contrast, the HOMA-IR as well as the plasma glucose, serum insulin and $\mathrm{HbA} 1 \mathrm{c}$ levels showed no significant differences between the three groups (Table 1). Meanwhile, the patients with an elevated $\operatorname{Lp}(\mathrm{a})$ level had a higher frequency of treatment with cardiac and antidiabetic medications, such as clopidogrel, vasodilators, diuretics and biguanide (Table 1), and a CochranMantel-Haenszel trend analysis comparing the $\mathrm{Lp}(\mathrm{a})$ tertile and extent of CAD revealed that the $\mathrm{Lp}(\mathrm{a})$ concentration displayed trend toward a positive association with the extent of CAD $(p<0.0001)$ (Table 2).

Over a mean follow-up period of $4.4 \pm 2.6$ years, there were 59 MACEs (35 cardiac deaths and 24 cases of non-fatal MI), with an event rate of $3.9 \%$. The respective MACE rates for the T1, T2 and T3 groups were $1.8 \%$ (9/499), $3.2 \%$ (16/497) and 6.8\% (34/498), respectively. According to the multivariate Cox proportional survival analysis, age (HR 1.064, 95\% CI 1.033-1.095, $p<0.0001$ ), a male gender (HR $1.889,95 \%$ CI 1.052-3.394, $p=0.033)$ and the extent of CAD $(p<0.0001)$ were independent risk factors for MACE (Table 3). Furthermore, the T3 group demonstrated an almost 3-fold higher risk of MACE compared to the T1 group (HR 2.890, 95\% CI 1.3736.084, $p=0.005$ ) (Table 3). In addition, the survival probability plot according to the $\mathrm{Lp}(\mathrm{a})$ tertile revealed that an elevated $\operatorname{Lp}(\mathrm{a})$ level is associated with a worse prognosis $(p=0.008)$ after adjusting for age, gender, hypertension, hyperlipidemia, smoking and the extent of CAD (Fig. 1).

In order to evaluate whether an elevated $\mathrm{Lp}(\mathrm{a})$ level has incremental prognostic value for MACE, statistical analyses, including assessments of the likelihood ratio, C-statistics, IDI, continuous NRI and NRI, were performed between model I (reference model consisting of age, gender, hypertension, smoking, hyperlipidemia and extent of CAD) and model II (model I with the addition of $\mathrm{Lp}(\mathrm{a})$ ) (Table 4). The respective likelihood ratios were 730.511 and 741.112 for models I and II, respectively, with a statistically 
Table 1. Baseline, laboratory and angiographic characteristics according to the $\mathrm{Lp}(\mathrm{a})$ tertile $(n=1,494)$

\begin{tabular}{|c|c|c|c|c|}
\hline \multirow[b]{2}{*}{$\begin{array}{l}\text { Median (range) } \\
\text { Number of patients }\end{array}$} & \multicolumn{3}{|c|}{ Lp(a) tertiles $(\mathrm{mg} / \mathrm{dl})$} & \multirow[b]{2}{*}{$p$-value } \\
\hline & $\begin{array}{c}\mathrm{T} 1 \\
4.7(0-8.5) \\
(n=499)\end{array}$ & $\begin{array}{c}\mathrm{T} 2 \\
13.5(8.6-20.7) \\
(n=497)\end{array}$ & $\begin{array}{c}\text { T3 } \\
38.8(20.8-218.0) \\
(n=498)\end{array}$ & \\
\hline Age (years) & $62.0 \pm 10.7$ & $63.9 \pm 9.7$ & $64.7 \pm 10.2$ & $<0.0001$ \\
\hline Male gender, $n(\%)$ & $336 / 499(67.3 \%)$ & $310 / 497(62.4 \%)$ & $285 / 498(57.2 \%)$ & 0.004 \\
\hline Hypertension, $n(\%)$ & $368 / 499(73.7 \%)$ & $358 / 497(72.0 \%)$ & $377 / 498(75.7 \%)$ & 0.420 \\
\hline Smoking, $n(\%)$ & $195 / 499(39.1 \%)$ & $150 / 497(30.2 \%)$ & $149 / 498(29.9 \%)$ & 0.002 \\
\hline Hyperlipidemia, $n(\%)$ & $307 / 499(61.5 \%)$ & $312 / 497(62.8 \%)$ & $339 / 498(68.1 \%)$ & 0.073 \\
\hline Clinical diagnosis & & & & 0.014 \\
\hline Silent IHD, $n(\%)$ & $103 / 499(20.6 \%)$ & $102 / 497(20.5 \%)$ & $106 / 498(21.3 \%)$ & \\
\hline Stable angina, $n(\%)$ & $245 / 499(49.1 \%)$ & $219 / 497(44.1 \%)$ & $186 / 498(37.3 \%)$ & \\
\hline Unstable angina, $n(\%)$ & $111 / 499(22.2 \%)$ & $130 / 497(26.2 \%)$ & $146 / 498(29.3 \%)$ & \\
\hline NSTEMI, $n(\%)$ & $25 / 499(5.0 \%)$ & $30 / 497(6.0 \%)$ & $32 / 498(6.4 \%)$ & \\
\hline STEMI, $n(\%)$ & $15 / 499(3.0 \%)$ & $16 / 497(3.2 \%)$ & $28 / 498(5.6 \%)$ & \\
\hline Obstructive CAD, $n(\%)$ & $349 / 499(69.9 \%)$ & $389 / 497(78.3 \%)$ & $435 / 498(87.3 \%)$ & $<0.0001$ \\
\hline Extent of CAD & & & & $<0.0001$ \\
\hline Non-obstructive CAD & $150 / 499(30.1 \%)$ & $108 / 497(21.7 \%)$ & $63 / 498(12.7 \%)$ & \\
\hline $1 \mathrm{VD}, n(\%)$ & $104 / 499(20.8 \%)$ & $97 / 497$ (19.5\%) & $94 / 498(18.9 \%)$ & \\
\hline 2VD, $n(\%)$ & $112 / 499(22.4 \%)$ & $116 / 497(23.3 \%)$ & $124 / 498(24.9 \%)$ & \\
\hline $3 \mathrm{VD}, n(\%)$ & $133 / 499(26.7 \%)$ & $176 / 497(35.4 \%)$ & $217 / 498(43.6 \%)$ & \\
\hline Revascularization, $n(\%)$ & $256 / 499(51.3 \%)$ & $297 / 497(59.8 \%)$ & $333 / 498(66.9 \%)$ & $<0.0001$ \\
\hline BMS, $n(\%)$ & $59 / 499(11.8 \%)$ & $95 / 498(19.1 \%)$ & $91 / 498(18.3 \%)$ & \\
\hline DES, $n(\%)$ & $157 / 499(31.5 \%)$ & $141 / 497(28.4 \%)$ & $169 / 498(33.9 \%)$ & \\
\hline $\mathrm{CABG}, n(\%)$ & $40 / 499(8.0 \%)$ & $61 / 497(12.3 \%)$ & 73/498 (14.7\%) & \\
\hline $\mathrm{Lp}(\mathrm{a})(\mathrm{mg} / \mathrm{dl})$ & $4.8 \pm 2.2$ & $13.9 \pm 3.5$ & $50.9 \pm 34.3$ & $<0.0001$ \\
\hline Total cholesterol $(\mathrm{mg} / \mathrm{dl})$ & $159.7 \pm 36.4$ & $163.3 \pm 37.6$ & $168.6 \pm 40.5$ & 0.001 \\
\hline Triglycerides $(\mathrm{mg} / \mathrm{dl})$ & $158.8 \pm 112.8$ & $143.8 \pm 81.7$ & $143.3 \pm 83.3$ & 0.012 \\
\hline LDL cholesterol (mg/dl) & $94.7 \pm 30.0$ & $97.7 \pm 31.1$ & $104.5 \pm 34.0$ & $<0.0001$ \\
\hline HDL cholesterol $(\mathrm{mg} / \mathrm{dl})$ & $41.8 \pm 11.5$ & $42.1 \pm 10.9$ & $41.7 \pm 11.4$ & 0.843 \\
\hline HbA1c (\%) & $7.58 \pm 1.42$ & $7.62 \pm 1.49$ & $7.68 \pm 1.50$ & 0.599 \\
\hline Glucose (mmol/L) & $7.28 \pm 2.30$ & $7.32 \pm 2.73$ & $7.48 \pm 2.89$ & 0.446 \\
\hline Insulin $(\mu \mathrm{IU} / \mathrm{mL})$ & $11.5 \pm 16.0$ & $11.0 \pm 10.8$ & $12.1 \pm 16.2$ & 0.499 \\
\hline HOMA-IR & $3.8 \pm 5.6$ & $3.6 \pm 3.9$ & $4.3 \pm 6.7$ & 0.125 \\
\hline \multicolumn{5}{|l|}{ Cardiac medications } \\
\hline Aspirin & $465 / 499(93.2 \%)$ & $465 / 497(93.6 \%)$ & $470 / 497(94.6 \%)$ & 0.648 \\
\hline Clopidogrel & $309 / 499(61.9 \%)$ & $333 / 497(67.0 \%)$ & $350 / 497(70.4 \%)$ & 0.017 \\
\hline Oral anticoagulants & $32 / 499(6.4 \%)$ & $23 / 497(4.6 \%)$ & $21 / 497(4.2 \%)$ & 0.247 \\
\hline Vasodilator & $273 / 499(54.7 \%)$ & $313 / 497(63.0 \%)$ & $334 / 497(67.2 \%)$ & $<0.0001$ \\
\hline$\beta$-blocker & $261 / 499(52.3 \%)$ & $298 / 497(60.0 \%)$ & $280 / 497(56.3 \%)$ & 0.051 \\
\hline ACE inhibitor & $152 / 499(30.5 \%)$ & $166 / 497(33.4 \%)$ & $170 / 497(34.2 \%)$ & 0.415 \\
\hline $\mathrm{ARB}$ & $279 / 499(55.9 \%)$ & $267 / 497(53.7 \%)$ & $285 / 497(57.3 \%)$ & 0.512 \\
\hline $\mathrm{CCB}$ & $234 / 499(46.9 \%)$ & $231 / 497(46.5 \%)$ & $223 / 497(44.9 \%)$ & 0.795 \\
\hline Diuretics & $109 / 499(21.8 \%)$ & $119 / 497(23.9 \%)$ & $142 / 497(28.6 \%)$ & 0.042 \\
\hline$\alpha$-blocker & $23 / 499(4.6 \%)$ & $22 / 497(4.4 \%)$ & $31 / 497(6.2 \%)$ & 0.360 \\
\hline \multicolumn{5}{|l|}{ Antidiabetic medications } \\
\hline$\alpha$-glucosidase inhibitor & $56 / 499(11.2 \%)$ & $69 / 497(13.9 \%)$ & $85 / 497(17.1 \%)$ & 0.028 \\
\hline Biguanide & $258 / 499(51.7 \%)$ & $224 / 497(45.1 \%)$ & $198 / 497(39.8 \%)$ & 0.001 \\
\hline Sulfonylurea/Glinide & $279 / 499(55.9 \%)$ & $256 / 497(51.5 \%)$ & $270 / 497(54.3 \%)$ & 0.369 \\
\hline Thiazolidinedione & $78 / 499(15.6 \%)$ & $77 / 497(15.5 \%)$ & $70 / 497(14.1 \%)$ & 0.752 \\
\hline DPP-4 inhibitor & $14 / 499(2.8 \%)$ & $11 / 497(2.2 \%)$ & $4 / 497(0.8 \%)$ & 0.063 \\
\hline Insulin therapy & $53 / 499(10.6 \%)$ & $74 / 497(14.9 \%)$ & $75 / 497(15.1 \%)$ & 0.066 \\
\hline
\end{tabular}

The data are expressed as numbers (\%) or the mean \pm standard deviation.

Abbreviations. $\mathrm{ACE}=$ angiotensin converting enzyme; $\mathrm{ARB}=$ angiotensin II receptor blocker; $\mathrm{CAD}=$ coronary artery disease; $\mathrm{CCB}=\mathrm{calcium}$ channel blocker; DPP-4= dipeptidyl peptidase-4; HDL=high-density lipoprotein; HOMA-IR=homeostasis model assessment insulin resistance index; IHD= ischemic heart disease; $\mathrm{LDL}=$ low-density lipoprotein; $\mathrm{Lp}(\mathrm{a})=$ lipoprotein $(\mathrm{a})$; NSTEMI = non-ST elevation myocardial infarction; STEMI $=S T$ elevation myocardial infarction; $\mathrm{T} 1=$ lower tertile; $\mathrm{T} 2=$ middle tertile; $\mathrm{T} 3=$ upper tertile; $\mathrm{VD}=$ vessel disease 
Table 2. Trend analysis between the Lp(a) level and extent of CAD

\begin{tabular}{lccc}
\hline & & Lp (a) tertiles & $p$-value \\
\cline { 2 - 4 } & T1 & T2 & T3 \\
\hline Non-obstructive CAD & $150 / 499(30.1 \%)$ & $108 / 497(21.7 \%)$ & $63 / 498(12.7 \%)$ \\
1VD, $n(\%)$ & $104 / 499(20.8 \%)$ & $97 / 497(19.5 \%)$ & $94 / 498(18.9 \%)$ \\
2VD, $n(\%)$ & $112 / 499(22.4 \%)$ & $116 / 497(23.3 \%)$ & $124 / 498(24.9 \%)$ \\
3VD, $n(\%)$ & $133 / 499(26.7 \%)$ & $176 / 497(35.4 \%)$ & $217 / 498(43.6 \%)$ \\
\hline
\end{tabular}

The data are expressed as numbers (\%).

Abbreviations. $\mathrm{CAD}=$ coronary artery disease; $\mathrm{Lp}(\mathrm{a})=$ lipoprotein $(\mathrm{a}) ; \mathrm{T} 1=$ lower tertile; $\mathrm{T} 2=$ middle tertile; $\mathrm{T} 3=$ upper tertile; $\mathrm{VD}=\mathrm{vessel}$ disease

Table 3. Univariate and multivariate Cox regression analysis for predicting MACE (non-fatal MI and cardiac death)

\begin{tabular}{|c|c|c|c|c|c|c|}
\hline & \multicolumn{3}{|c|}{ Univariate } & \multicolumn{3}{|c|}{ Multivariate } \\
\hline & HR & $95 \% \mathrm{CI}$ & $p$-value & HR & $95 \% \mathrm{CI}$ & $p$-value \\
\hline Age & 1.068 & $1.038-1.100$ & $<0.0001$ & 1.064 & $1.033-1.095$ & $<0.0001$ \\
\hline Male gender & 1.688 & $0.951-2.998$ & 0.074 & 1.889 & $1.052-3.394$ & 0.033 \\
\hline Hypertension & 1.324 & $0.715-2.451$ & 0.372 & & & \\
\hline Smoking & 0.883 & $0.511-1.525$ & 0.655 & & & \\
\hline Hyperlipidemia & 0.883 & $0.521-1.497$ & 0.644 & & & \\
\hline Obstructive CAD & 3.322 & $1.203-9.177$ & 0.021 & & & \\
\hline Extent of CAD & & & $<0.0001$ & & & $<0.0001$ \\
\hline 1VD & 0.733 & $0.164-3.276$ & 0.684 & 0.534 & $0.119-2.401$ & 0.413 \\
\hline 2VD & 1.191 & $0.336-4.223$ & 0.787 & 0.847 & $0.237-3.022$ & 0.798 \\
\hline 3VD & 6.223 & $2.237-17.306$ & $<0.0001$ & 3.541 & $1.246-10.065$ & 0.018 \\
\hline $\operatorname{Lp}(\mathrm{a})$ & & & $<0.0001$ & & & 0.008 \\
\hline T2 & 1.849 & $0.817-4.186$ & 0.140 & 1.542 & $0.679-3.503$ & 0.300 \\
\hline T3 & 3.869 & $1.855-8.067$ & $<0.0001$ & 2.890 & $1.373-6.084$ & 0.005 \\
\hline Revascularization & 0.908 & $0.537-1.535$ & 0.718 & & & \\
\hline
\end{tabular}

Abbreviations. $\mathrm{CAD}=$ coronary artery disease; $\mathrm{CI}=$ confidence interval; $\mathrm{HR}=$ hazard ratio; $\mathrm{Lp}(\mathrm{a})=\operatorname{lipoprotein}(\mathrm{a})$; $\mathrm{MACE}=$ major adverse cardiac event; $\mathrm{MI}=$ myocardial infarction; $\mathrm{T} 2=$ middle tertile; $\mathrm{T} 3=$ upper tertile; $\mathrm{VD}=$ vessel disease

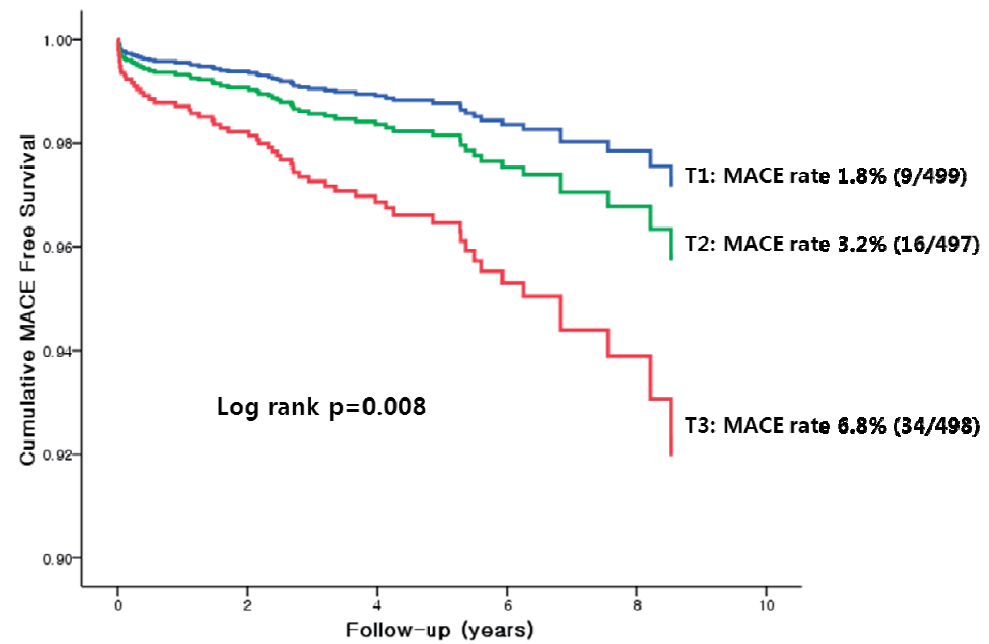

Fig. 1. Survival probability plots according to the $\mathrm{Lp}(\mathrm{a})$ tertile after adjusting for age, gender, hypertension, smoking, hyperlipidemia and the extent of CAD

Abbrevviations. $\operatorname{Lp}(\mathrm{a})=$ lipoprotein $(\mathrm{a}) ; \mathrm{MACE}=$ major adverse cardiac event; $\mathrm{T} 1=$ lower tertile; $\mathrm{T} 2=$ middle tertile; $\mathrm{T} 3=$ upper tertile 
Table 4. Incremental prognostic value of $L p(a)$ for predicting MACE

\begin{tabular}{|c|c|c|c|c|}
\hline & Model I & Model II & Difference & $p$-value \\
\hline Likelihood ratio & 730.531 & 741.112 & 10.581 & 0.005 \\
\hline C-statistics & $0.7817(0.7153-0.8481)$ & $0.7926(0.7288-0.8564)$ & $0.0108(-0.0144-0.0362)$ & 0.400 \\
\hline Relative IDI & \multicolumn{2}{|c|}{$0.0216(0.008-0.0409)$} & - & $<0.001$ \\
\hline Continuous NRI & \multicolumn{2}{|c|}{$0.5721(0.0867-0.9565)$} & - & 0.012 \\
\hline NRI & \multicolumn{2}{|c|}{$0.1549(0.0241-0.3514)$} & - & 0.004 \\
\hline
\end{tabular}

$95 \%$ confidence intervals are in parentheses.

Model I: age, gender, hypertension, smoking, hyperlipidemia and extent of CAD; Model II: Model I + Lp(a)

Abbreviations. IDI = integrated discrimination improvement; $\mathrm{Lp}(\mathrm{a})=\operatorname{lipoprotein}(\mathrm{a}) ; \mathrm{MACE}=$ major adverse cardiac event; NRI = net reclassification improvement

significant difference of 10.581 between the two models $(p=0.005)$. The respective C-statistics were 0.7817 and 0.7926 for models I and II, respectively, for a difference of 0.0108 between the two models, without statistical significance $(p=0.400)$. However, an elevated $\mathrm{Lp}(\mathrm{a})$ level was found to have incremental prognostic value for predicting MACE in terms of the IDI $(2.16 \%$ improvement, $p<0.001)$, continuous NRI (57.21\% improvement, $p=0.012)$ and NRI $(15.49 \%$ improvement, $p=0.004)$, when comparing the two models (Table 4).

\section{Discussion}

We evaluated the prognostic value of an elevated $\mathrm{Lp}$ (a) level in type 2 diabetic patients with symptomatic CAD. The principal findings in this study are that: 1) the $\operatorname{Lp}(\mathrm{a})$ level exhibits a positive correlation with the extent as well as presence of obstructive CAD in type 2 diabetic patients; 2) an elevated $\mathrm{Lp}(\mathrm{a})$ level is an independent risk factor for MACE in type 2 diabetic patients with symptomatic CAD; 3 ) an elevated $\mathrm{Lp}(\mathrm{a})$ level is associated with a worse prognosis in type 2 diabetic patients with symptomatic CAD; and 4) an elevated $\mathrm{Lp}(\mathrm{a})$ level has incremental prognostic value for MACE in type 2 diabetic patients with symptomatic CAD. Taken together, our findings revealed the value of an elevated $\mathrm{Lp}(\mathrm{a})$ level in type 2 diabetic patients with symptomatic CAD.

Patients with type 2 diabetes present with a metabolic profile different from that of the general population and display a cardiovascular risk two- to fourfold higher than non-diabetic individuals ${ }^{1,2)}$. In addition, an elevated $\mathrm{Lp}(\mathrm{a})$ level is considered to have a continuous, independent and modest association with the development of cardiovascular diseases in the general population ${ }^{5}$. Both of these cardiovascular risk factors (type 2 diabetes and $\mathrm{Lp}(\mathrm{a})$ ) are crucial parameters that should be assessed when managing patients at risk for the development of cardiovascular disease. Intrigu- ingly, Mora et al. demonstrated that the Lp(a) level is independently and inversely associated with the risk of type 2 diabetes $^{7)}$. As that study consisted of the cohorts from the Women's Health Study and Copenhagen City Heart Study, for a total of 36,398 patients, it is now generally accepted that the $\mathrm{Lp}(\mathrm{a})$ levels are lower in type 2 diabetic patients than in non-diabetic patients ${ }^{11,12)}$. However, this finding does not signify that an elevated $\mathrm{Lp}$ (a) level is not attributable to cardiovascular disease in type 2 diabetic patients.

Previous studies have reported that the serum insulin level has a negative association with the $\mathrm{Lp}(\mathrm{a})$ level ${ }^{13,14)}$. Moreover, another study revealed that the $\mathrm{Lp}$ (a) level is inversely related to metabolic syndrome and its components, suggesting that the insulin level and insulin sensitivity are factors disturbing the assessment of $\operatorname{Lp}(\mathrm{a})^{15}$. In the current study, biguanides were less frequently used in the elevated Lp(a) group, which may have influenced the insulin sensitivity in type 2 diabetic patients. However, there were no significant statistical differences in the HbA1c, plasma glucose, serum insulin or HOMA-IR levels between the three groups.

Several studies have highlighted the impact of an elevated $\mathrm{Lp}(\mathrm{a})$ level in type 2 diabetic patients with or without $\mathrm{CAD}^{16,17)}$. One study reported that type 2 diabetic patients with CAD have higher $\mathrm{Lp}(\mathrm{a})$ levels than both type 2 diabetic patients without CAD and control subjects (non-diabetic patients without CAD $)^{16)}$. Another study reported that type 2 diabetic patients with CAD have lower Lp(a) levels than nondiabetic patients with $\mathrm{CAD}^{17)}$. However, that study also revealed that type 2 diabetic patients with CAD have higher $L p(a)$ levels than type 2 diabetic patients without $\mathrm{CAD}^{17)}$. Cross-sectional studies have previously reported that type 2 diabetic patients with coronary heart disease have higher $\mathrm{Lp}(\mathrm{a})$ levels than those without coronary heart disease ${ }^{6,18,19)}$. In addition, several studies have reported a positive association between an elevated Lp(a) level and CAD among type 
2 diabetic patients ${ }^{16,20,21)}$. In accordance with these studies, our findings demonstrated that an elevated $\mathrm{Lp}(\mathrm{a})$ level has a positive correlation with the extent as well as presence of obstructive CAD in type 2 diabetic patients.

The prognostic value of elevated $\mathrm{Lp}(\mathrm{a})$ in type 2 diabetic patients remains a subject under dispute. Previous studies in general populations have documented that an elevated $\mathrm{Lp}(\mathrm{a})$ level is associated with a worse prognosis, ${ }^{511}$. Additionally, subgroup analyses of the type 2 diabetic cohorts in these same studies showed that an elevated $\mathrm{Lp}(\mathrm{a})$ level is associated with a worse outcome ${ }^{5,11}$. Studies of type 2 diabetic patients have also demonstrated that an elevated $\operatorname{Lp}(\mathrm{a})$ level is a risk factor for cardiovascular disease ${ }^{22,23)}$. In contrast, several studies have suggested that an elevated $\operatorname{Lp}(\mathrm{a})$ has no association with the development of cardiovascular disease in type 2 diabetic patients ${ }^{24-26)}$. In the context of these conflicting findings, we evaluated the prognostic value of an elevated Lp(a) level in type 2 diabetic patients with symptomatic CAD. In the current study, we found an elevated Lp(a) level to be associated with a worse outcome in type 2 diabetic patients with symptomatic CAD, even after adjusting for other confounding risk factors. We believe that our data support the clinical value of an elevated $\mathrm{Lp}(\mathrm{a})$ level in type 2 diabetic patients.

The inconsistent results regarding the prognostic value of elevated $\mathrm{Lp}(\mathrm{a})$ in type 2 diabetic patients may be due to differences in the follow-up period, study cohort size, characteristics of the study cohort (age, gender, ethnicity), duration of type 2 diabetes and $\mathrm{Lp}$ (a) assay method, as mentioned by $\mathrm{Qi}$ et al. ${ }^{24)}$. Therefore, a larger cohort-based prospective study is warranted to clarify the prognostic value of an elevated $\mathrm{Lp}$ (a) level in type 2 diabetic patients.

Although several of the aforementioned studies have revealed that an elevated $\mathrm{Lp}(\mathrm{a})$ level is associated with worse cardiovascular outcomes, none have discriminated the incremental prognostic value of elevated $L p(a)$ in terms of the prognosis ${ }^{5,11,22,23)}$. To our knowledge, this is the first study to demonstrate the incremental prognostic value of an elevated Lp(a) level in type 2 diabetic patients with symptomatic CAD. Our findings suggest that an elevated Lp(a) level may contribute to an increased risk of cardiovascular disease in type 2 diabetic patients and that the $\mathrm{Lp}(\mathrm{a})$ level is an important clinical consideration, not only in the general population, but also patients with type 2 diabetes.

There are several limitations associated with the present study. First, this was an observational study. Second, the study was performed at a single center in
Korea and consisted only of Korean patients. Since the $\mathrm{Lp}$ (a) levels vary according to race and ethnicity, it is uncertain whether our results are equally applicable in general clinical practice.

In conclusion, an elevated Lp(a) level is associated with worse outcomes in type 2 diabetic patients with symptomatic CAD, and an elevated Lp(a) level has incremental prognostic value in this patient population.

\section{Acknowledgements}

This study was supported by the Brain Korea 21 Project for Medical Science, Yonsei University and the Korean Institute of Medicine.

\section{Conflicts of Interest}

None.

\section{References}

1) Beckman JA, Creager MA, Libby P: Diabetes and atherosclerosis. JAMA, 2002; 287: 2570-2581

2) Almdal T, Scharling H, Jensen JS, Vestergaard H: The independent effect of type 2 diabetes mellitus on ischemic heart disease, stroke, and death: a population-based study of 13000 men and women with 20 years of follow-up. Arch Intern Med, 2004; 164: 1422-1426

3) Berglund L, Ramakrishnan R: Lipoprotein(a): an elusive cardiovascular risk factor. Arterioscler Thromb Vasc Biol, 2004; 24: 2219-2226

4) Danesh J, Collins R, Peto R: Lipoprotein(a) and coronary heart disease: meta-analysis of prospective studies. Circulation, 2000; 102: 1082-1085

5) Erqou S, Kaptoge S, Perry PL, Di AE, Thompson A, White IR, Marcovina SM, Collins R, Thompson SG, Danesh J: Lipoprotein(a) concentration and the risk of coronary heart. JAMA, 2009; 302: 412-423

6) Murase T, Okubo M, Amemiya-Kudo M, Ebara T, Mori Y: Impact of elevated serum lipoprotein(a) concentrations on the risk of coronary heart disease in patients with type 2 diabetes mellitus. Metabolism, 2008; 57: 791-795

7) Mora S, Kamstrup PR, Rifai N, Nordestgaard BG, Buring JE, Ridker PM: Lipoprotein(a) and risk of type 2 diabetes. Clin Chem, 2010; 56: 1252-1260

8) Kwon SW, Lee BK, Hong BK, Kim JY, Choi EY, Sung JM, Rhee JH, Park YM, Ma DW, Chung H, Mun HS, Lee SJ, Park JK, Min PK, Yoon YW, Rim SJ, Kwon HM: Prognostic significance of elevated lipoprotein(a) in coronary artery revascularization patients. Int J Cardiol, 2013; 167: 1990-1994

9) Pencina MJ, D'Agostino RB Sr., D'Agostino RB Jr., Vasan RS: Evaluating the added predictive ability of a new marker: From area under the ROC curve to reclassification and beyond. Stat Med, 2008; 27: 157-172

10) Pencina MJ, D’Agostino RB Sr., Steyerberg EW: Exten- 
sions of net reclassification improvement calculations to measure usefulness of new biomarkers. Stat Med, 2011; 30: 11-21

11) Kamstrup PR, Benn M, Tybjaerg-Hansen A, Nordestgaard BG: Extreme lipoprotein(a) levels and risk of myocardial infarction in the general population: the Copenhagen City Heart Study. Circulation, 2008; 117: 176-184

12) Ridker PM, Cook NR, Lee IM, Gordon D, Gaziano JM, Manson JE, Hennekens CH, Buring JE: A randomized trial of low-dose aspirin in the primary prevention of cardiovascular disease in women. N Engl J Med, 2005; 352: 1293-1304

13) Rainwater DL, Haffner SM: Insulin and 2-hour glucose levels are inversely related to $\mathrm{Lp}(\mathrm{a})$ concentrations controlled for LPA genotype. Arterioscler Thromb Vasc Biol, 1998; 18: 1335-1341

14) Chien KL, Lee YT, Sung FC, Su TC, Hsu HC, Lin RS: Lipoprotein (a) level in the population in Taiwan: relationship to sociodemographic and atherosclerotic risk factors. Atherosclerosis, 1999; 143: 267-273

15) Sung KC, Wild SH, Byrne CD: Lipoprotein (a), metabolic syndrome and coronary calcium score in a large occupational cohort. Nutr Metab Cardiovasc Dis, 2013; 23: $1239-1246$

16) Mohan V, Deepa R, Haranath SP, Premalatha G, Rema M, Sastry NG, Enas EA: Lipoprotein(a) is an independent risk factor for coronary artery disease in NIDDM patients in South India. Diabetes Care, 1998; 21: 18191823

17) Gazzaruso C, Garzaniti A, Falcone C, Geroldi D, Finardi G, Fratino P: Association of lipoprotein(a) levels and apolipoprotein(a) phenotypes with coronary artery disease in Type 2 diabetic patients and in non-diabetic subjects. Diabet Med, 2001; 18: 589-594

18) James RW, Boemi M, Sirolla C, Amadio L, Fumelli P, Pometta D: Lipoprotein (a) and vascular disease in dia- betic patients. Diabetologia, 1995; 38: 711-714

19) Ruiz J, Thillet J, Huby T, James RW, Erlich D, Flandre P, Froguel P, Chapman J, Passa P: Association of elevated lipoprotein(a) levels and coronary heart disease in NIDDM patients. Relationship with apolipoprotein(a) phenotypes. Diabetologia, 1994; 37: 585-591

20) Watts GF, ap Gwilym RM, Mazurkiewicz J, Coltart J: Independent correlation between plasma lipoprotein(a) and angiographic coronary artery disease in NIDDM. Diabetes Care, 1995; 18: 234-236

21) Pedreño J, Fernández R, Ballester A, Jornet A, Usón M, Canela J, Petit M: Lack of association of serum lipoprotein (a) levels with type-2 diabetes mellitus in patients with angiographically defined coronary artery disease. Int J Cardiol, 2000; 74: 159-167

22) Hiraga T, Kobayashi T, Okubo M, Nakanishi K, Sugimoto T, Ohashi Y, Murase T: Prospective study of lipoprotein(a) as a risk factor for atherosclerotic cardiovascular disease in patients with diabetes. Diabetes Care, 1995; 18: 241-244

23) Hernández C, Francisco G, Chacón P, Simó R: Lipoprotein(a) as a risk factor for cardiovascular mortality in type 2 diabetic patients: a 10-year follow-up study. Diabetes Care, 2005; 28: 931-933

24) Qi Q, Workalemahu T, Zhang C, Hu FB, Qi L: Genetic variants, plasma lipoprotein(a) levels, and risk of cardiovascular morbidity and mortality among two prospective cohorts of type 2 diabetes. Eur Heart J, 2012; 33: 325334

25) Abu-Lebdeh HS, Hodge DO, Nguyen TT: Predictors of macrovascular disease in patients with type 2 diabetes mellitus. Mayo Clin Proc, 2001; 76: 707-712

26) Saely CH, Koch L, Schmid F, Marte T, Aczel S, Langer P, Hoefle G, Drexel H: Lipoprotein(a), type 2 diabetes and vascular risk in coronary patients. Eur J Clin Invest, 2006; 36: 91-97 